Rev. Int. Contam. Ambie. 35 (1) 125-135, 2019

DOI: 10.20937/RICA.2019.35.01.09

\title{
CAMBIOS EN LA ACTIVIDAD DE ENZIMAS ANTIOXIDANTES DE Myriophyllum quitense EXPUESTA A ENDOSULFÁN A DIFERENTES TEMPERATURAS
}

\author{
Daniela Soledad GARANZINI*, Fernando Gastón ITURBURU y Mirta Luján MENONE
}

Instituto de Investigaciones Marinas y Costeras, Universidad Nacional de Mar del Plata-Consejo Nacional de Investigaciones Científicas y Técnicas, Funes 3350, Mar del Plata, Buenos Aires, Argentina

*Autora para correspondencia; dgaranzi@mdp.edu.ar

(Recibido octubre 2017; aceptado abril 2018)

Palabras clave: plaguicida, estrés oxidativo, macrófita acuática, factores de confusión

\section{RESUMEN}

Las macrófitas acuáticas han sido ampliamente utilizadas como biomonitores ambientales por su rol primordial en el ecosistema y porque los cuerpos de agua son el destino final de muchos xenobióticos. En particular, se han utilizado varios biomarcadores del género Myriophyllum para evaluaciones de toxicidad; sin embargo, existen pocos antecedentes sobre el efecto potencial de los "factores de confusión" sobre dichos biomarcadores. El objetivo del presente trabajo fue evaluar el efecto de la temperatura en la actividad de enzimas antioxidantes en Myriophyllum quitense expuesta a concentraciones de relevancia ambiental del plaguicida endosulfán (ES). Se evaluó la actividad de las enzimas catalasa (CAT) y guayacol peroxidasa (POD), así como glutatión-S-transferasa en su fracción citosólica (GSTc) y microsomal (GSTm), en plantas expuestas a dos temperaturas $\left(11\right.$ y $\left.19^{\circ} \mathrm{C}\right)$ y a dos concentraciones de ES: 5 y $10 \mu \mathrm{g} / \mathrm{L}$. Se observó interacción entre la temperatura y la concentración de ES para las actividades de CAT y POD, y diferencias en las actividades de las cuatro enzimas cuando las plantas fueron expuestas a $11^{\circ} \mathrm{C}$, respecto de los controles. Sin embargo, no se observaron diferencias significativas en las actividades enzimáticas de las plantas expuestas a ES a $19^{\circ} \mathrm{C}$. Estos resultados indican la importancia de considerar la evaluación de factores como la temperatura en bioensayos, dado que su efecto altera la toxicidad del contaminante.

Keywords: pesticide, oxidative stress, aquatic macrophyte, confounding factors

\begin{abstract}
Aquatic macrophytes have been widely used as environmental biomonitors because of their primary role in ecosystems and because water bodies are the final destination of many xenobiotics. Particularly, several biomarkers have been used in the genus Myriophyllum for toxicity tests. However, there is scarce evidence of the potential effect of the "confounding factors" on these biomarkers. The objective of the present work was to evaluate the possible effect of temperature on the activity of antioxidant enzymes in Myriophyllum quitense exposed to environmentally relevant concentrations of the insecticide endosulfan. The activity of the enzymes catalase (CAT) and guaiacol peroxidase (POD), as well as glutathione-s-transferase in the cytosolic (GSTc) and
\end{abstract}


microsomal (GSTm) fractions, were evaluated in plants exposed to two temperatures: 11 and $19^{\circ} \mathrm{C}$, and two concentrations of endosulfan (ES): 5 and $10 \mu \mathrm{g} / \mathrm{L}$. Interaction between temperature and ES concentration for CAT and POD activities was observed. Differences in the activities of all enzymes were detected when plants were exposed to $1{ }^{\circ} \mathrm{C}$ with respect to controls. However, no significant differences were observed in the enzymatic activities of the plants exposed to ES at $19{ }^{\circ} \mathrm{C}$. These results emphasize the importance of considering the evaluation of factors such as temperature in bioassays, since its effect alters the toxicity of the contaminant.

\section{INTRODUCCIÓN}

En los últimos años, los principales países productores de materias primas han incrementado la actividad agrícola a partir del empleo de insumos externos y tecnología, permitiendo su extensión a tierras que no habían sido cultivables hasta entonces (Viglizzo et al. 2011).

El uso de las zonas no cultivables ha generado mayor vulnerabilidad en las zonas litorales de los cuerpos de agua adyacentes y en los organismos que allí habitan (Melzer 1999), debido a que estos cuerpos son susceptibles a recibir y acumular contaminantes de origen agrícola (Jergentz et al. 2004, Wendt-Rasch et al. 2004).

Los contaminantes presentes en la columna de agua pueden ser incorporados por plantas o animales y magnificarse en la cadena trófica, lo que constituye un riesgo potencial para la salud humana y ambiental (Lytle y Lytle 2001).

$\mathrm{El}$ endosulfán (ES, $\mathrm{C}_{9} \mathrm{H}_{6} \mathrm{Cl}_{6} \mathrm{O}_{3} \mathrm{~S}$ ) es un plaguicida de amplio espectro, perteneciente al grupo de los organoclorados, que se utiliza para el control de numerosos insectos en cultivos (Sohn et al. 2004, Di Marzio et al. 2005). Como consecuencia de su uso se ha detectado en atmósfera, cuerpos de agua, sedimentos y biota (Menone et al. 2001, Sethunathan et al. 2002, Jergentz et al. 2004, González et al. 2012).

El ES tiene un tiempo de vida media en agua de aproximadamente un mes $\left(20^{\circ} \mathrm{C}, \mathrm{pH}: 7\right)$ (de Lorenzo et al. 2002), siendo considerable su presencia en los cuerpos de agua dulce, ya que puede llegar allí por escorrentía de los suelos donde el compuesto fue aplicado. Se han encontrado concentraciones en este medio de hasta $100 \mu \mathrm{g} / \mathrm{L}$ (Mersie et al. 2003).

Niveles del orden de magnitud mencionados previamente suponen un riesgo para la salud de los organismos acuáticos, ya que de acuerdo con la Agencia de Protección Ambiental de Estados Unidos (USEPA, por sus siglas en inglés), valores superiores a 0.22 y $0.056 \mu \mathrm{g} / \mathrm{L}$ generarían un impacto agudo y crónico, respectivamente (Mersie et al. 2003).
La Convención de Estocolmo de 2012 prohibió el uso de ES junto con otros compuestos orgánicos persistentes; sin embargo, en algunos países como Argentina se han obtenido permisos para seguir aplicándolos por un tiempo adicional hasta acabar con los remanentes disponibles (Amaya 2011).

Así, Silva Barni (2017) informa valores de ES de 0.004 a $0.017 \mu g / L$ en aguas del río Quequén Grande, Provincia de Buenos Aires, en el periodo de muestreo 2014-2015, a pesar de haber transcurrido varios años desde su prohibición.

Un biomarcador se define como el cambio en una respuesta biológica (desde molecular hasta comportamental, pasando por la celular y fisiológica) que se pueden relacionar con la exposición a los efectos tóxicos de químicos ambientales (Peakall 1994).

Cuando las respuestas de los biomarcadores se desvían del rango normal - es decir, de una situación sin estrés-, superando determinado umbral de concentración de contaminante o tiempo de exposición, se genera una situación de efectos múltiples a niveles jerárquicos de organización biológica (ORNL 1991, Brain y Cedergreen 2008, Pagano et al. 2016).

Entonces, la utilización de biomarcadores evidencia que el compuesto se ha incorporado en un organismo, se ha distribuido en sus tejidos y ha originado un efecto en determinados sitios blanco (Brain y Cedergreen 2008).

Los biomarcadores deben cumplir con ciertos criterios para considerarse como tales: a) ser sensibles; b) responder al tóxico en forma dependiente de la dosis o el tiempo, lo que permite determinar la magnitud de la exposición o el efecto; c) ser relativamente fáciles de medir, y d) contemplar falsos negativos y falsos positivos (Huggett et al. 1992, van der Oost et al. 2003).

Los falsos resultados pueden generarse por la presencia de factores no relacionados con la contaminación que interfieren en las respuestas esperadas. Estos "factores de confusión en la interpretación de la toxicidad" (Den Besten y Munawar 2005) pueden ser fisiológicos y ambientales, tales como la edad, 
el sexo, la condición reproductiva, la temperatura, la disponibilidad del alimento, el $\mathrm{pH}$ y la salinidad, entre otros (Jiménez et al. 1990, Vidal et al. 2002).

La presencia de factores de confusión debe tenerse en cuenta en los ensayos desarrollados en laboratorio para poder validarlos en campo, de modo que si se comparan las respuestas de organismos de diferentes sitios contaminados y de referencia, los organismos deben recolectarse de forma tal que tanto las condiciones fisiológicas como las variables ambientales sean similares en los diferentes sitios (ORNL 1991).

En macrófitas se ha demostrado que, por ejemplo, la disponibilidad de nutrientes y su limitación pueden modificar las tasas de crecimiento y en consecuencia la sensibilidad de las pruebas de toxicidad (Brooks et al. 2015).

En las plantas, las especies reactivas del oxígeno (ERO) son producidas constantemente y se localizan en diferentes compartimentos celulares como producto de varias rutas metabólicas (Foyer y Mullineaux 1994); en algunos casos cumplen con la función de ser moléculas de señalización, mediando la respuesta a diferentes estímulos (Neill et al. 2002).

El mantenimiento de la homeostasis de las especies reactivas es esencial para la salud fisiológica de los organismos (Ames et al. 1975). Cuando existe un desbalance entre la producción de especies moleculares oxidantes y antioxidantes, encontrándose en mayor proporción las primeras, la condición se denomina estrés oxidativo (Boelsterli 2007, Gill y Tuteja 2010).

Existen muchos biomarcadores que se emplean para la evaluación del estrés oxidativo. Dentro de éstos se encuentran los de efecto (van der Oost et al. 2003), que incluyen enzimas antioxidantes como la catalasa (CAT), las peroxidasas (PX) y la glutatiónS-transferasa (GST), así como compuestos no enzimáticos, como el glutatión o el ascorbato, y los que dañan la membrana lipídica, como el producto de peroxidación lipídica malondialdehído (MDA).

Las enzimas CAT y guayacol peroxidasa (POD) tienen como principal función detoxificar $\mathrm{H}_{2} \mathrm{O}_{2}$, mientras que la enzima GST posee dos roles principales: la catálisis de reacciones de conjugación con el tripéptido glutatión en fase II de metabolismo, tanto animal como vegetal, y la participación en la defensa contra el estrés oxidativo mediante la detoxificación directa de peróxidos lipídicos de membranas en vegetales (Marrs 1996, Cummins et al. 1999).

Los organismos fotosintéticos poseen características ventajosas respecto del uso de animales, por lo que han sido ampliamente utilizados para el monitoreo de metales, herbicidas, surfactantes y compuestos clorados (Nimptsch et al. 2005). El género Myriophyllum (familia Haloragaceae) (Darlington y Wylie 1961), una hierba acuática flotante sumergida y arraigada por sus raíces al sedimento, común en orillas de lagos y ríos, es cosmopolita.

Este género ha sido ampliamente utilizado para evaluaciones de toxicidad de plaguicidas (Turgut et al. 2003, Nimptsch et al. 2005, Nimptsch y Pflugmacher 2004, Menone et al. 2008) y está presente en ambos hemisferios, siendo Myriophyllum spicatum el preponderante en el hemisferio norte y Myriophyllum quitense (Kunth) en el hemisferio sur (Orchard 1981).

En la literatura se encuentran disponibles referencias de exposición en animales a múltiples sustancias y en particular a ES. Sin embargo, este mismo tipo de resultados es escaso cuando se emplean plantas como organismos modelo.

Ramachandran et al. (1984) evaluaron tasas fotosintéticas y respiratorias de seis macrófitas expuestas durante $6 \mathrm{~h}$ a ES; encontraron una disminución de la fotosíntesis en diferente medida, dependiendo de las especies evaluadas. Por otro lado, de Lorenzo et al. (2002) determinaron que la $\mathrm{EC}_{50}$ de Pseudokirchneriella subcapitata, medida como la tasa de crecimiento, es de $427.80 \mu \mathrm{g} / \mathrm{L}$.

Se han informado otros efectos, como daño en el material genético observado como fragmentación del ADN, mediante el ensayo cometa en Trifolium repens L. (Liu et al. 2009), y como incremento en la frecuencia de aberraciones cromosómicas en anafase- telofase (ACAT) en Bidens laevis (Pérez et al. 2008, 2011).

Pérez et al. (2014) evaluaron el efecto de varias concentraciones de ES y distintos tiempos de exposición, y hallaron un aumento en la frecuencia de ACAT en plantas expuestas durante $48 \mathrm{~h}$ a $10 \mu \mathrm{g} / \mathrm{L}$ ES, así como aumento en la actividad de la enzima POD y disminución de la actividad de GST microsomal (GSTm).

En este contexto, el objetivo del presente trabajo fue evaluar si diferentes temperaturas pueden modificar la actividad de enzimas antioxidantes en $M$. quitense expuesta a concentraciones de relevancia ambiental del plaguicida endosulfán.

\section{MATERIALES Y MÉTODOS}

\section{Organismos}

Los ejemplares de Myriophyllum quitense se obtuvieron en reservorios naturales del sudeste de la provincia de Buenos Aires (Argentina), y se generaron 
nuevos individuos mediante reproducción vegetativa. Se aclimataron en el laboratorio en acuarios de $30 \mathrm{~L}$ acondicionados con sustrato artificial y medio nutritivo Hoagland puro (Hoagland y Arnon 1950). Se mantuvieron durante 15 días con fotoperiodo natural 10:14 (luz: oscuridad) y a temperaturas de $11 \mathrm{o} 19^{\circ} \mathrm{C}$, según correspondiese.

\section{Soluciones experimentales}

Las soluciones de exposición del plaguicida endosulfán se prepararon a partir de soluciones madre de los isómeros alfa y beta del compuesto puro $(6,7,8,9,10,10$-hexacloro-1,5,5a,6,9a,9-hexahidro-6,9-metano-2,4,3-benzo(e)dioxatiepina-3-óxido, Sigma), respetando la proporción 70:30 (alfa:beta) que poseen los formulados comerciales.

Como control negativo se utilizó una solución de medio Hoagland que contenía una alícuota del disolvente dimetilsulfóxido (DMSO), a una concentración final de $0.004 \%$. Trabajos previos realizados con $M$. quitense han demostrado que esta concentración de DMSO no afecta las actividades de las enzimas evaluadas debido a que no se hallaron diferencias significativas entre un control con medio Hoagland y DMSO $(0.004 \%)$ y un control sólo con medio Hoagland (Menone et al. 2008)

Las plantas fueron expuestas a dos concentraciones de endosulfán $(5$ y $10 \mu \mathrm{g} / \mathrm{L})$, durante $24 \mathrm{~h}$, utilizándose cinco muestras independientes para cada tratamiento $(\mathrm{N}=5)$. Las temperaturas se eligieron debido a que recrean las condiciones que habría en el ambiente natural, cuyas temperaturas promedio son de $9{ }^{\circ} \mathrm{C}$ en invierno y $22{ }^{\circ} \mathrm{C}$ en verano (Cruz y Escalante 2001).

\section{Preparación de extractos enzimáticos}

Para la preparación de los extractos y la medición de la actividad enzimática se siguió la metodología de Pflugmacher (2004). Se molieron con $\mathrm{N}_{2}$ líquido, en mortero, $5 \mathrm{~g}$ de tejido húmedo. Se agregaron $10 \mathrm{~mL}$ de solución amortiguadora sodio-fosfato $(0.1 \mathrm{M}$, pH: 6.5) con glicerol $20 \%(\mathrm{v} / \mathrm{v})$, DTE $14 \mathrm{mM}$ y EDTA $1 \mathrm{mM}$.

Se removieron los restos celulares por centrifugación $\left(10000 \mathrm{~g}, 4^{\circ} \mathrm{C}, 10 \mathrm{~min}\right)$. El sobrenadante se centrifugó (100 $\left.000 \mathrm{~g}, 4^{\circ} \mathrm{C}, 60 \mathrm{~min}\right)$ para separar la fracción citosólica de la asociada a membrana. El precipitado, que corresponde a la fracción microsomal, se resuspendió en $1 \mathrm{~mL}$ de solución amortiguadora microsomal (sodio-fosfato $20 \mathrm{mM}, \mathrm{pH}$ : 7.0, glicerol $20 \%$ y DTE $1.4 \mathrm{mM}$ ) y se conservó en congelador a $-80{ }^{\circ} \mathrm{C}$ (previo congelamiento con $\mathrm{N}_{2}$ líquido) hasta su posterior análisis.
Se cuantificó el volumen del sobrenadante y se agregó sulfato de amonio en una concentración de $35 \%$ de saturación, permaneciendo en agitación constante durante $1 \mathrm{~h}$ sobre hielo. Se centrifugó (20000 g, $4{ }^{\circ} \mathrm{C}, 20 \mathrm{~min}$ ), se descartó el precipitado y se agregó sulfato de amonio al sobrenadante a una saturación del $80 \%$, permaneciendo en agitación constante durante 1.5 hora sobre hielo.

Se centrifugó $\left(30000 \mathrm{~g}, 4{ }^{\circ} \mathrm{C}, 30 \mathrm{~min}\right)$ y el precipitado que contenía las proteínas solubles se resuspendió en solución amortiguadora sodio-fosfato (20 mM, pH: 7). El extracto se desaló por filtración en gel con columnas NAP-10 (GE-Healthcare, UK), conservándose en congelador $\mathrm{a}-80{ }^{\circ} \mathrm{C}$ hasta la medición, previo congelamiento con $\mathrm{N}_{2}$ líquido.

La actividad enzimática se evaluó por duplicado con un espectrofotómetro.

\section{Mediciones enzimáticas}

Para cuantificar la actividad de la enzima GST se utilizó como sustrato CDNB (1-cloro-2,4-dinitrobenceno) siguiendo la metodología de Habig et al. (1974). La enzima CAT se evaluó mediante la disminución de $\mathrm{H}_{2} \mathrm{O}_{2}$, utilizando el protocolo de Greenwald (1985). La actividad de POD se llevó a cabo según el protocolo de Bergmeyer (1974), utilizando guayacol como sustrato de la enzima.

La actividad enzimática se expresó como nanokatales por miligramo de proteína (nkat/mg), donde un katal corresponde a la conversión de un mol de sustrato por segundo. La cantidad de proteína presente en la muestra se midió por el método de Bradford (1976), empleando una curva de calibración de albúmina bovina.

\section{Análisis estadístico}

Todos los datos presentaron normalidad (según la prueba de Shapiro-Wilk) y fueron analizados con la prueba de análisis de varianza (ANDEVA) de dos factores, para evaluar si existía interacción entre los factores "temperatura" y concentraciones de ES.

La interacción se visualiza en la figura 1 como la intersección de las líneas, lo cual significa que la variable dependiente (e.g., la actividad enzimática de POD) es influenciada por estos dos factores. Cuando no existe interacción se observan líneas paralelas que se interpretan como efectos sobre la variable dependiente de un factor o del otro.

Luego, los datos se analizaron mediante la comparación de las concentraciones de ES respecto del control negativo que fue expuesto a la misma temperatura empleando la prueba de ANDEVA de una vía para analizar diferencias entre las medias y una 
prueba de Tukey para establecer qué concentraciones de ES mostraban diferencias respecto al control negativo (Zar 1996). En todos los casos el valor de significancia fue del $5 \%$.

\section{RESULTADOS}

Los valores basales (control negativo) de las enzimas GSTc, GSTm, CAT y POD resultaron similares a ambas temperaturas de exposición, 11 y $19^{\circ} \mathrm{C}$ $(\mathrm{p}>0.05)$. Las pruebas de ANOVA de dos factores realizados para cada uno de los biomarcadores enzimáticos mostraron efecto de la interacción entre las variables "temperatura" y "ES" en las enzimas CAT y POD (p < 0.05; Fig. 1a, b).

En el caso de las enzimas GSTm y GSTc no hubo efecto de interacción de los tratamientos $(\mathrm{p}>0.05$; Fig. 1c, d). Sin embargo, las cuatro enzimas evaluadas mostraron el efecto del tratamiento ES cuando la temperatura de exposición fue de $11^{\circ} \mathrm{C}$, mientras que a $19^{\circ} \mathrm{C}$ las plantas expuestas a ES no mostraron diferencias respecto de las plantas control.
A $11{ }^{\circ} \mathrm{C}$ las actividades de CAT y POD disminuyeron en plantas expuestas a ambas concentraciones de ES ( $p<0.05$, Figs. 2 y 3 , respectivamente), mientras que a $19{ }^{\circ} \mathrm{C}$ no se observó diferencia significativa respecto de los controles ( $p>0.05$, Figs. 2 y 3 ).

En ambas fracciones, la actividad de la enzima GST no mostró diferencias significativas respecto al control cuando las plantas fueron expuestas a ES y la temperatura fue de $19{ }^{\circ} \mathrm{C}(\mathrm{p}>0.05)$. Por otra parte, en plantas expuestas a $11^{\circ} \mathrm{C}$ se observó un incremento significativo de la actividad de GSTc a la concentración de $10 \mu \mathrm{g} / \mathrm{L}$ ES ( $<<0.05$, Fig. 4), y de GSTm a $5 \mu \mathrm{g} / \mathrm{L}$ ES ( $<00.05$, Fig. 5).

\section{DISCUSIÓN}

En el presente trabajo, los cuatro biomarcadores bioquímicos evaluados en $M$. quitense presentaron cambios en sus respuestas a la exposición a ES dependientes de la temperatura, mostrando diferencias de actividad únicamente cuando la temperatura fue de $11^{\circ} \mathrm{C}$.
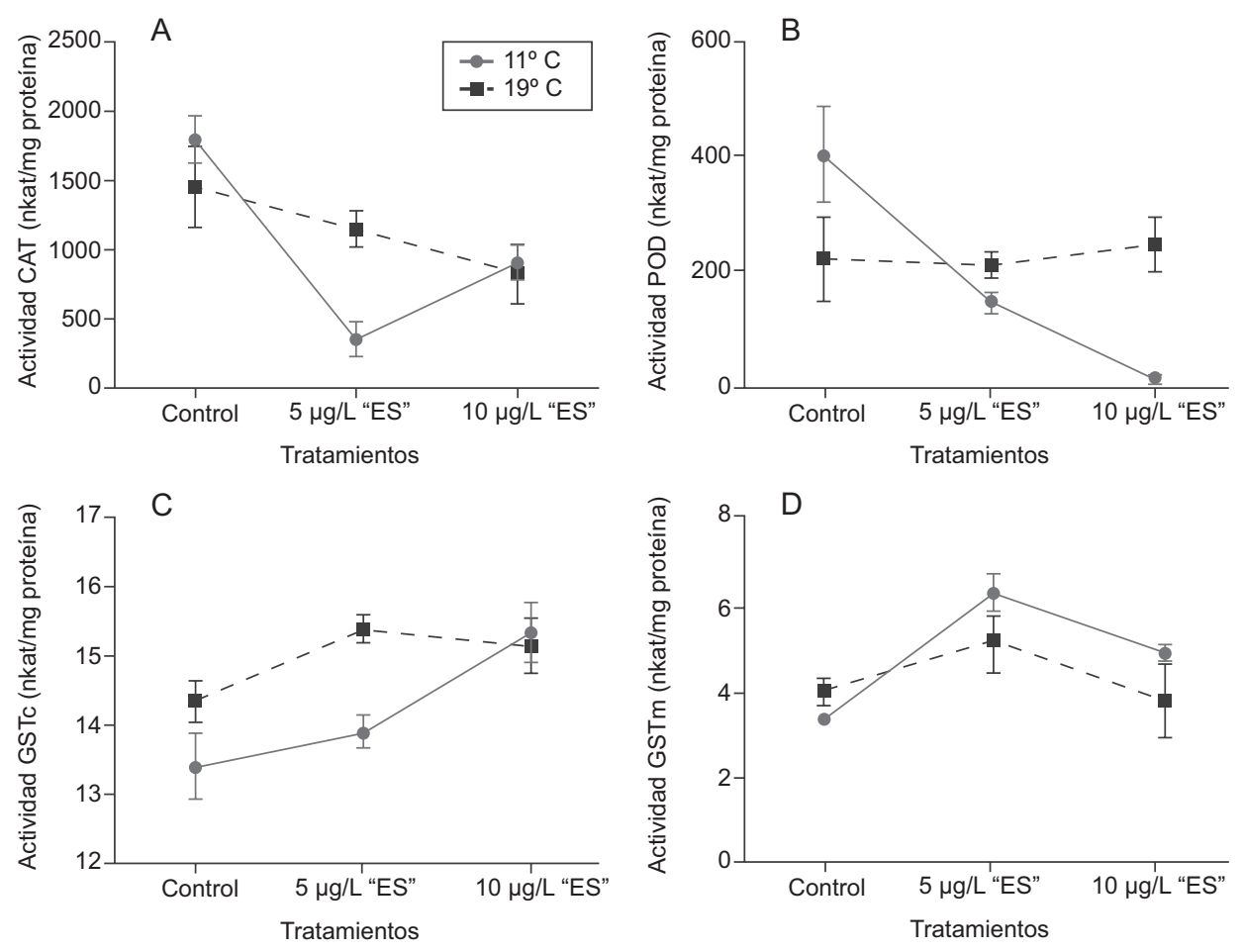

Fig. 1. Análisis de interacción de la temperatura y la concentración de endosulfán sobre actividades enzimáticas de Myriophyllum quitense. (a) Catalasa (CAT), (b) guaiacol peroxidasa (POD), (c) glutatión-S-transferasa citosólico (GSTc), (d) glutatión-S-transferasa microsomal (GSTm). $\mathrm{N}=5$ 


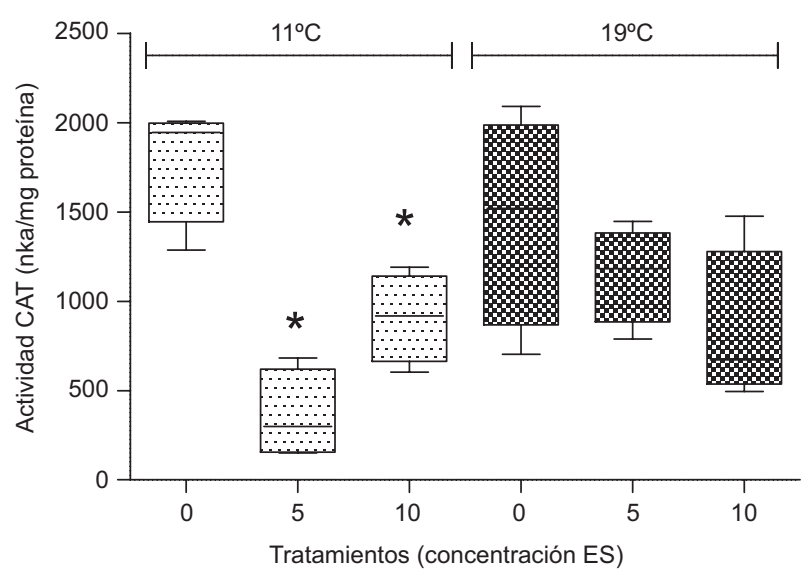

Fig. 2. Actividad de catalasa (CAT) (nkat/mg proteína) en Myriophyllum quitense expuesta a distintas temperaturas. Cajas y bigotes: media \pm error estándar de la media. *Indica diferencias significativas respecto del control (tratamiento correspondiente a $0 \mathrm{mg} / \mathrm{L} \mathrm{ES}$ ) (prueba de Tukey) ( $\mathrm{p}<0.05) . \mathrm{N}=5$

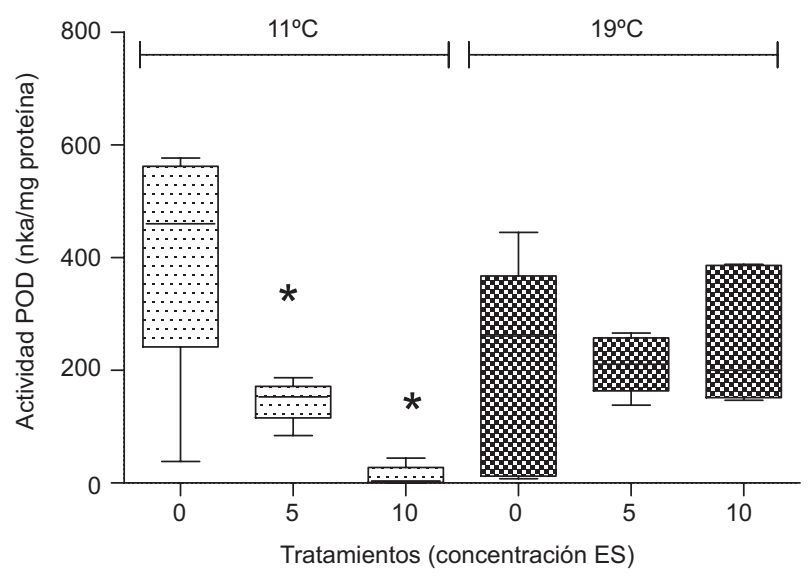

Fig. 3. Actividad de guayacol peroxidasa (POD) (nkat/mg proteína) en Myriophyllum quitense expuesta a distintas temperaturas. Cajas y bigotes: media \pm error estándar de la media. *Indica diferencias significativas respecto del control (tratamiento correspondiente a $0 \mathrm{mg} / \mathrm{L} \mathrm{ES}$ ) (prueba de Tukey) $(\mathrm{p}<0.05) . \mathrm{N}=5$

Por ello es relevante tener en cuenta la temperatura en ensayos de laboratorio con $M$. quitense. Las enzimas evaluadas presentaron niveles de actividad semejantes en las plantas expuestas a control a diferentes temperaturas de exposición $\left(11\right.$ y $\left.19^{\circ} \mathrm{C}\right)$, lo cual indica que la temperatura por sí sola no modifica estos parámetros, siendo ventajoso respecto de otros parámetros como la tasa de crecimiento y la capacidad fotosintética, los cuales se ha demostrado que sí dependen de la temperatura (Tasmin et al. 2014a), adjudicándose entonces una modificación en las actividades enzimáticas al xenobiótico.

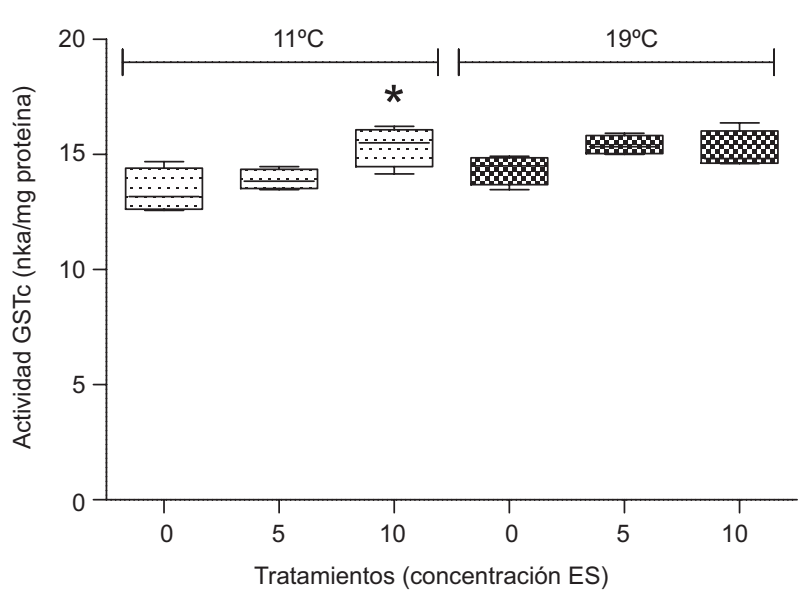

Fig. 4. Actividad de glutation-S-transferasa citosólica (GSTc) (nkat/mg proteína) en Myriophyllum quitense expuesta a distintas temperaturas. Cajas y bigotes: media \pm error estándar de la media. *Indica diferencias significativas respecto del control (tratamiento correspondiente a 0 $\mathrm{mg} / \mathrm{L}$ ES) (prueba de Tukey) $(\mathrm{p}<0.05) . \mathrm{N}=5$

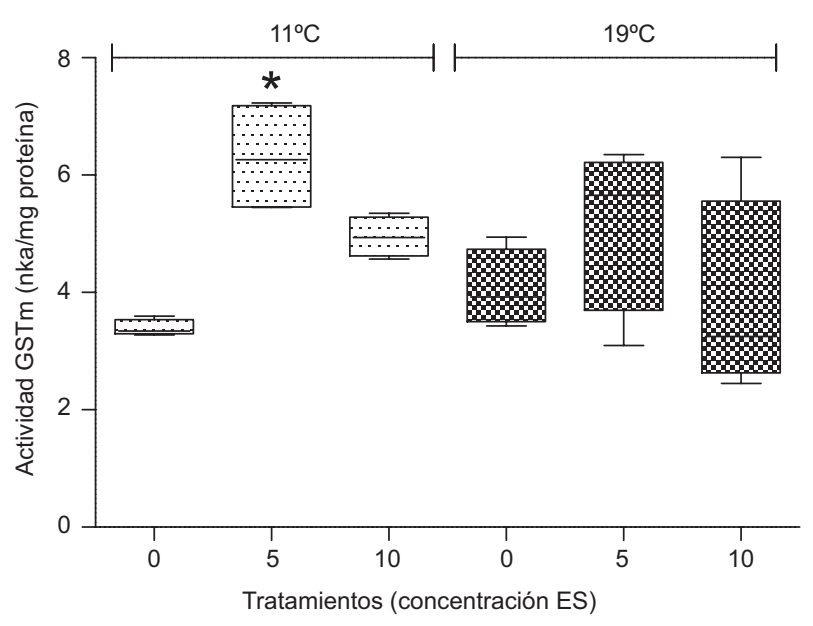

Fig. 5. Actividad de glutation-S-transferasa microsomal (GSTm) (nkat/mg proteína) en Myriophyllum quitense expuesta a distintas temperaturas. Cajas y bigotes: media \pm error estándar de la media. *Indica diferencias significativas respecto del control (tratamiento correspondiente a 0 $\mathrm{mg} / \mathrm{L}$ ES) (prueba de Tukey) $(\mathrm{p}<0.05) . \mathrm{N}=5$

Esta respuesta constante de los controles resulta significativa si se considera que en los criterios establecidos para el uso de biomarcadores en estudios de campo se incluye el conocimiento y comprensión de la variabilidad debida a factores ambientales (Huggett et al. 1992).

Pflugmacher et al. (2000) evaluaron la actividad basal de GST en ambas fracciones, con diferentes sustratos en 59 especies vegetales. Estos estudios mostraron que, en general, la actividad de GSTm 
fue menor que la actividad de GSTc para una misma especie, tal como se observa para M. quitense en los controles negativos del presente trabajo.

Sin embargo, la sensibilidad al ES fue mayor en la GSTm, debido a que mostró una actividad incrementada a $5 \mu \mathrm{g} / \mathrm{L}$ ES. Esta mayor sensibilidad podría explicarse por la especificidad de las múltiples isoformas de GST involucradas en la respuesta a xenobióticos, la cual se ha demostrado que es especieespecífica y tiene como blanco un amplio espectro de compuestos electrofílicos (Coleman et al. 1997, Fujimiya et al. 2016).

La actividad de la enzima GST en ambas fracciones aumentó cuando las plantas fueron expuestas a ES y la temperatura de exposición fue de $11^{\circ} \mathrm{C}$, mostrando la participación de este sistema antioxidante en la respuesta al efecto de estrés oxidativo que se ha informado previamente para el ES en $M$. quitense (Menone et al. 2008).

Estos autores, sin embargo, encontraron un aumento en la actividad de GSTc a $5 \mu \mathrm{g} / \mathrm{L}$ en $M$. quitense expuesta a ES en contradicción con los resultados de este trabajo, lo que podría deberse a condiciones de ensayo diferentes, ya que en dicho ensayo la temperatura de exposición fue de $20-22^{\circ} \mathrm{C}$. Esta discrepancia apoya la hipótesis de que en $M$. quitense las enzimas tienen una actividad diferencial cuando están expuestas a un xenobiótico, dependiente de la temperatura del medio.

La enzima CAT es la principal enzima detoxificadora de $\mathrm{H}_{2} \mathrm{O}_{2}$, aunque también posee la capacidad de oxidar otros sustratos como metanol, etanol, formaldehído y ácido fórmico (van Breusegem et al. 2001).

Por otra parte, las peroxidasas pertenecen a una familia enzimática ubicua que también cataliza la reducción de $\mathrm{H}_{2} \mathrm{O}_{2}$, y oxida numerosos sustratos y compuestos fenólicos (Mehlhorn et al. 1996). La enzima POD sólo se encuentra presente en plantas (Asada 1992); utiliza compuestos fenólicos como cosustratos y su actividad se relaciona con condiciones ambientales adversas, contribuyendo a la actividad antioxidante (Michałowicz et al. 2009).

Ambas enzimas se han estudiado previamente en M. quitense. Nimptsch et al. (2005) encontraron que la actividad de POD aumentó en plantas expuestas a metales pesados. Menone et al. (2008) evaluaron la actividad de CAT expuesta a ES y hallaron incrementos significativos. Posteriormente, Garanzini y Menone (2015) encontraron una disminución en la actividad de ambas enzimas cuando las plantas fueron expuestas a $50 \mu \mathrm{g} / \mathrm{L}$ del fungicida azoxistrobina.

Los resultados obtenidos por Menone et al. (2008) indican un proceso de estrés oxidativo en presencia de ES, observado a través del incremento de la actividad de enzimas antioxidantes simultáneamente al incremento en el contenido de $\mathrm{H}_{2} \mathrm{O}_{2}$.

Sin embargo, los resultados aquí expuestos muestran una disminución en la actividad de estas enzimas en presencia de ES, que sería generada por una condición hiperoxidativa capaz de inactivar la actividad enzimática y, consecuentemente, conduciría a perder la capacidad antioxidante y en última instancia a la muerte celular (Connell 2005).

El hecho de que no se observen cambios en las actividades de las enzimas evaluadas en plantas expuestas a endosulfán respecto a las no expuestas, cuando la temperatura fue de $19^{\circ} \mathrm{C}$, podría deberse a un incremento en la tasa metabólica basal a esta temperatura.

Dicho incremento implicaría una mayor tasa de procesos anabólicos y catabólicos, incluyendo una mayor actividad enzimática basal, es decir, los organismos tendrían una mayor actividad per se debido al efecto de la temperatura. Esta explicación fue propuesta por otros autores respecto del incremento de procesos de detoxificación y excreción de xenobióticos en moluscos acuáticos (Greco et al. 2011).

El hecho de que no se observen cambios significativos en las actividades enzimáticas en plantas expuestas a ES, con relación a las de control, a una temperatura de $19{ }^{\circ} \mathrm{C}$ - como sí ocurre a los 11 ${ }^{\circ} \mathrm{C}$ - pone de manifiesto que las plantas expuestas a mayor temperatura estarían en un estado fisiológico favorable, mientras que a temperaturas bajas se alejan de esta condición óptima y la presencia de ES genera estrés.

Si bien hay pocos trabajos que evalúan el efecto de factores de confusión en la toxicidad de plaguicidas en organismos fotosintéticos, en términos generales los resultados sugieren que los efectos adversos, y en algunos casos la toxicidad, se manifiestan a temperaturas bajas, mientras que son menores a temperaturas elevadas debido a una actividad enzimática basal mayor.

Los parámetros que se evalúan en los trabajos antes citados no suelen estar vinculados con sistemas de alarma temprana, como actividades de enzimas antioxidantes; sino a evaluaciones de punto final relacionadas con el crecimiento (e.g., crecimiento total, contenido de pigmentos y contenido de $\mathrm{H}_{2} \mathrm{O}_{2}$ ).

Así, Tasmin et al. (2014a) evaluaron en Pseudokirchenriella subcapitata el efecto de la temperatura y las concentraciones de nitratos y sulfatos en el porcentaje de inhibición de crecimiento combinado con el posible efecto del herbicida diurón. En dicho 
trabajo, los autores informaron una tasa mayor de crecimiento a mayores temperaturas, así como disminución de la toxicidad del herbicida debida a un aumento en la temperatura.

Por otra parte, Tasmin et al. (2014b) evaluaron también el efecto del herbicida diurón en el crecimiento total del organismo antes citado a diferentes temperaturas, así como el porcentaje de inhibición fotosintético y el contenido de $\mathrm{H}_{2} \mathrm{O}_{2}$ en $P$. subcapitata. En este estudio encontraron que organismos expuestos al herbicida a temperaturas más bajas presentaban mayor contenido de $\mathrm{H}_{2} \mathrm{O}_{2}$, mayor tasa de inhibición de eficiencia fotosintética y menor tasa de crecimiento.

Ambos trabajos se realizaron con tiempos de exposición menores a 144 h. Así, de manera similar a los resultados obtenidos en el presente trabajo, la temperatura puede modificar la toxicidad de un compuesto, y sus efectos dependerán tanto de la especie evaluada como de la sustancia a la que se la expone (Cairns et al. 1975).

Hall et al. (1997) evaluaron el efecto en macrófitas de la exposición subcrónica al herbicida atrazina en Potamogeton pectinatus, teniendo en cuenta distintos niveles de salinidad del agua y hallaron que este factor generó modificaciones en la toxicidad del herbicida evaluado.

Por ello la evaluación de biomarcadores debe tener en cuenta estos factores, sobre todo cuando son evaluados en campo, para evitar una mala interpretación de la toxicidad. Por ello

resulta de interés comprender la influencia de dichos factores de confusión sobre las respuestas a xenobióticos en organismos no blanco, como M. quitense. Esta especie ha mostrado que es sensible ante la exposición a ES, y dicha sensibilidad es diferente según la temperatura evaluada.

Considerando las fluctuaciones de temperatura en el ambiente de aplicación, éste debería ser un parámetro fundamental al realizar evaluaciones de riesgo ambiental (ERA), debido a que la simple evaluación de exposición a contaminantes podría arrojar resultados no representativos de su efecto en condiciones reales, subestimando el riesgo de exposición.

Finalmente, teniendo en cuenta que estudios previos realizados en organismos fotosintéticos han mostrado una sensibilidad diferencial a ciertos plaguicidas ante variaciones de temperatura, debe tenerse en mente que los organismos fotosintéticos comprenden la mayor parte de la biósfera (Maluszynska y Juchimiuk 2005).

Por otra parte, los contaminantes presentes en la columna de agua pueden ser incorporados por plantas o animales y magnificarse en la cadena trófica, constituyendo un riesgo potencial para la salud humana y ambiental (Lytle y Lytle 2001). Por ello

son necesarios más estudios con macrófitas como organismos biomonitores, considerando las variaciones en factores de confusión como temperatura, $\mathrm{pH}$ del agua, concentración de nutrientes, disponibilidad de oxígeno, calidad y cantidad de luz, entre otros.

\section{CONCLUSIONES}

El ES produce estrés oxidativo en células de $M$. quitense, puesto en evidencia a través del incremento de actividad de la enzima GST y la disminución de las actividades de CAT y POD. Por otra parte, se concluye que los efectos del plaguicida ES en $M$. quitense fueron influenciados por las diferentes temperaturas del medio ensayadas, ya que las respuestas enzimáticas fueron diferentes según la temperatura fuese de 11 o $19^{\circ} \mathrm{C}$.

En este sentido cobra importancia evaluar factores como la temperatura en los bioensayos, dado que son un factor de confusión al momento de interpretar los resultados.

\section{AGRADECIMIENTOS}

Los autores agradecen al Fondo para la Investigación Científica y Tecnológica y a la Universidad Nacional de Mar del Plata, por financiar este proyecto (Proyecto PICT 20131348 y Proyecto EXA795/16, respectivamente), y a la Dra. Andrea Crupkin por su asistencia en los análisis estadísticos.

\section{REFERENCIAS}

Amaya J.E. (2011). Resolución 511-2011 Servicio Nacional de Sanidad y Calidad Agroalimentaria (SENASA) [en línea]. http://www.senasa.gob.ar/normativas/ resolucion-511-2011-senasa-servicio-nacional-desanidad-y-calidad-agroalimentaria 09/04/2018

Ames B.N., McCann J. y Yamasaki E. (1975). Methods for detecting carcinogens and mutagens with the Salmonella/mammalian-microsome mutagenicity test. Mutat. Res. Envir. Muta. 31 (6), 347-363. DOI: 10.1016/0165-1161(75)90046-1

Asada K. (1992). Ascorbate peroxidase -a hydrogen peroxide-scavenging enzyme in plants. Physiol. Plantarum 85 (2), 235-241.

DOI: 10.1111/j.1399-3054.1992.tb04728.x 
Bergmeyer H.U. (1974). Methods of ezymatic analysis. $2^{\mathrm{a}}$ ed. Academic Press, Nueva York, EUA, pp. 685-690.

Boelsterli U.A. (2007). Mechanistic toxicology: The molecular basis of how chemicals disrupt biological targets. $2^{\mathrm{a}}$ ed. CRC Press, Taylor and Francis Group, Boca Ratón, Florida, EUA, 399 pp.

Bradford M.M. (1976). A rapid and sensitive method for the quantitation of microgram quantities of protein utilizing the principle of protein-dye binding. Anal. Biochem. 72 (1), 248-254.

DOI: $10.1016 / 0003-2697(76) 90527-3$

Brain R.A. y Cedergreen N. (2008). Biomarkers in aquatic plants: Selection and utility. En: Reviews of environmental contamination and toxicology (de Voogt P., Ed.). Springer, Nueva York, EUA, pp. 49-109.

DOI: 10.1007/978-0-387-09647-6_2

Brooks B.W., Fulton B.A. y Hanson M.L. (2015). Aquatic toxicology studies with macrophytes and algae should balance experimental pragmatism with environmental realism. Sci. Total Environ. 536, 406-407.

DOI: 10.1016/j.scitotenv.2015.07.085

Cairns Jr. J., Heath A.G. y Parker B.C. (1975). The effects of temperature upon the toxicity of chemicals to aquatic organisms. Hydrobiologia 47 (1), 135-171. DOI: 10.1007/BF00036747

Coleman J.O.D., Blake-Kajff M.M.A. y Davies T.G.E. (1997). Detoxification of xenobiotics by plants: chemical modification and vacuolar compartmentation. Trends Plant. Sci. 2 (4), 144-151. DOI: 10.1016/S1360-1385(97)01019-4

Connell D.W. (2005). Basic concepts of environmental chemistry. $2^{\mathrm{a}}$ ed. CRC Press, Taylor and Francis Group, Boca Ratón, Florida, EUA, 480 pp.

Cummins I., Cole D.J. y Edwards R. (1999). A role for glutathione transferases functioning as glutathione peroxidases in resistance to multiple herbicides in black grass. Plant J. 18 (3), 285-292.

DOI: 10.1046/j.1365-313X.1999.00452.x

Cruz M. y Escalante A.H. (2001). Effect of temperature on copepod species growth and development time: an experimental study. Memorias. Aquatic Sciences Meeting, American Society of Limnology and Oceanography (ASLO). Alburquerque, Nuevo Mexico, EUA, 12 al 16 de febrero, pp. 41. Darlington C.D. y Wylie A.P. (1961). Chromosome atlas of flowering plants. Allen y Umwin, Londres, Reino Unido, 519 pp.

De Lorenzo M.E., Taylor L.A., Lund S.A., Pennington P.L., Strozier E.D. y Fulton M.H. (2002). Toxicity and bioconcentration potencial of the agricultural pesticide endosulfan in phytoplankton and zooplancton. Arch. Environ. Con. Tox. 42 (2), 173-181.

DOI: $10.1007 / \mathrm{s} 00244-001-0008-3$
Den Besten P.J. y Munawar M. (2005). Ecotoxicological testing of marine and freshwater ecosystems: emerging techniques, trends, and strategies. CRC Press Taylor and Francis, Boca Ratón, Florida, EUA, 271 pp.

DOI: 10.1201/9781420037500.fmatt

Di Marzio W., Sáenz E., Alberdi J., Tortorelli M., Nannini P. y Ambrini G. (2005). Bioaccumulation of endosulfan from contaminated sediment by Vallisneria spiralis. B. Environ. Contam. Tox. 74 (4), 637-644.

DOI: $10.1007 / \mathrm{s} 00128-005-0631-1$

Foyer C.H. y Mullineaux P. (1994). Causes of photooxidative stress and amelioration of defense systems in plants. CRC Press Inc., Boca Ratón, Florida, EUA, $395 \mathrm{pp}$.

Fujimiya T., Kobayashi Y., Aoki T., Kohyama N., Ohbayashi M., Murakami M. y Yamamoto T. (2016). Effect of the GSTM1 null genotype on glutathione s- transferase (gst) activity in patients with non-viral liver tumors. J. Showa Med. Sci. 28 (2), 113-121. DOI: $10.15369 /$ sujms.28.113

Garanzini D.S. y Menone M.L. (2015). Azoxystrobin causes oxidative stress and DNA damage in the aquatic macrophyte Myriophyllum quitense. B. Environ. Contam. Tox. 94 (2), 146-151.

DOI: $10.1007 / \mathrm{s} 00128-014-1428-\mathrm{x}$

Gill S.S. y Tuteja N. (2010). Reactive oxygen species and antioxidant machinery in abiotic stress tolerance in crop plants. Plant Physiol. Bioch. 48 (12), 909-930. DOI:10.1016/j.plaphy.2010.08.016

González M., Miglioranza K.S., Shimabukuro V.M., Londoño O.M.Q., Martinez D.E., Aizpún J.E. y Moreno V.J. (2012). Surface and groundwater pollution by organochlorine compounds in a typical soybean system from the south Pampa, Argentina. Environ. Earth Sci. 65 (2), 481-491. DOI: 10.1007/s12665-011-1328-x

Greco L., Pellerin J., Capri E., Garnerot F., Louis S., Fournier M., Sacchi A., Fusi M., Lapointe D. y Couture P. (2011). Physiological effects of temperature and herbicide mixture on the soft-shell clam Mya arenaria (Mollusca, Bivalvia). Environ. Toxicol. Chem. 30 (1), 132-141. DOI: 10.1002/etc.359

Greenwald R.A. (1985). CRC handbook of methods for oxygen radical research. $3^{\mathrm{a}}$ ed. CRC Press, Boca Ratón, Florida, EUA, 447 pp.

Habig W.H., Pabst M.J. y Jakoby W.B. (1974). Glutathione S-transferases, the first enzymatic step in mercapturic acid formation. J. Biol. Chem. 249 (22), 7130-7139.

Hall Jr. L.W., Anderson R.D. y Ailstock M.S. (1997). Chronic toxicity of atrazine to sago pondweed at a range of salinities: implications for criteria development and ecological risk. Arch. Environ. Con. Tox. 33 (3), 261-267.

DOI: $10.1007 / \mathrm{s} 002449900252$ 
Hoagland D. y Arnon D. (1950). The water-culture method for growing plants without soil. California Agricultural Experiment Station. The College of Agriculture, University of California. Circular $\mathrm{N}^{\circ} 347$. Berkeley, California, EUA, 32 pp.

Huggett R.J., Kimerle R.A., Mehrle Jr. P.M. y Bergman H.L. (1992). Biomarkers. biochemical and physiological markers of anthropogenic stress. Lewis, Chelsea, Inglaterra, $347 \mathrm{pp}$.

Jergentz S., Mugni H., Bonetto C. y Schulz R. (2004). Runoff-related endosulfan contamination and aquatic macroinvertebrate response in rural basins near Buenos Aires, Argentina. Arch. Environ. Con. Tox. 46 (3), 345-352.

DOI: $10.1007 / \mathrm{s} 00244-003-2169-8$

Jiménez C., Niell F.X. y Fernández J.A. (1990). The photosynthesis of Dunaliella parva Lerche as a function of temperature, light and salinity. Hydrobiologia 197 (1), 165-172. DOI: $10.1007 /$ BF00026948

Lytle J.S. y Lytle T.F. (2001). Use of plants for toxicity assessment of estuarine ecosystems. Environ. Toxicol. Chem. 20 (1), 68-83. DOI: 10.1002/etc.5620200107

Liu W., Zhu L.S., Wang J., Wang J.H., Xie H. y Song Y. (2009). Assessment of the genotoxicity of endosulfan in earthworm and white clover plants using the comet assay. Arch. Environ. Con. Tox. 56 (4), 742-746.

DOI: $10.1007 / \mathrm{s} 00244-009-9309-8$

Maluszynska J. y Juchimiuk J. (2005). Plant genotoxicity: A molecular cytogenetic approach in plant bioassays. Arch. Ind. Hyg. Toxicol. 56 (2), 177-184.

Marrs K.A. (1996). The functions and regulation of glutathione S-transferases in plants. Annu. Rev. Plant Biol. 47 (1), 127-158.

DOI: 10.1146/annurev.arplant.47.1.127

Mehlhorn H., Lelandais M., Korth H. y Foyer C. (1996). Ascorbate is the natural substrate for plant peroxidases. FEBS Lett. 378 (3), 203-206. DOI: 10.1016/0014-5793(95)01448-9

Melzer A. (1999). Aquatic macrophytes as tools for lake management. Hydrobiologia 395 (0), 181-190.

DOI: 10.1023/A:1017001703033

Menone M.L., Aizpún de Moreno J.E., Moreno V.J., Lanfranchi A.L., Metcalfe T.L. y Metcalfe C.D. (2001). Organochlorine pesticides and PCBs in a Southern Atlantic coastal lagoon watershed, Argentina. Arch. Environ. Con. Tox. 40 (3), 355-362.

DOI: $10.1007 / \mathrm{s} 002440010183$

Menone M.L., Pesce S.F., Díaz M.P., Moreno J.D. y Wunderlin D.A. (2008). Endosulfan induces oxidative stress and changes on detoxication enzymes in the aquatic macrophyte Myriophyllum quitense. Phytochemistry 60 (5), 1150-1157.

DOI: 10.1016/j.phytochem.2007.11.016
Mersie W., Seybold C.A., McNamee C. y Lawson M.A. (2003). Abating endosulfan from runoff using vegetative filter strips: The importance of plant species and flow rate. Agr. Ecosyst. Environ. 97 (1-3), 215-223. DOI: $10.1016 / \mathrm{S} 0167-8809(03) 00035-5$

Michałowicz J., Urbanek H., Bukowska B. y Duda W. (2009). Chloroguaiacols change some antioxidative parameters and affect the activity of glutathione Stransferase in the leaves of reed Canary Grass (Phalaris arudinacea). Water Air Soil Pollut. 207 (1-4), 19-28. DOI: $10.1007 / \mathrm{s} 11270-009-0115-8$

Neill S., Desikan R. y Hancock J. (2002). Hydrogen peroxide signalling. Curr. Opin. Plant Biol. 5 (5), 388-395. DOI: 10.1016/S1369-5266(02)00282-0

Nimptsch J., Wunderlin D.A., Dollan A. y Pflugmacher S. (2005). Antioxidant and biotransformation enzymes in Myriophyllum quitense as biomarkers of heavy metal exposure and eutrophication in Suquía River basin (Córdoba, Argentina). Chemosphere 61 (2), 147-157. DOI: 10.1016/j.chemosphere.2005.02.079

Nimptsch J. y Pflugmacher S. (2005). Substrate specificities of cytosolic glutathione-S transferases in five different species of the aquatic macrophyte Myriophyllum. J. Appl. Bot. Food Qual. 79 (2), 94- 99.

Orchard A.E. (1981). A revision of South American Myriophyllum (Haloragaceae), and its repercussions on some Australian and North American species. Brunonia 4 (1), 27-65. DOI: 10.1071/BRU9810027

ORNL (1991). Conceptual strategy for design, implementation, and validation of a biomarker-based biomonitoring capability. Reporte técnico. Oak Ridge National Laboratory, Washington, EUA, 85 pp.

Pagano L., Servín A.D., de La Torre-Roche R., Mukherjee A., Majumdar S., Hawthorne J., Marmiroli M., Maestri E., Marra R.E., Isch S.M., Dhankher O.P., White J.C. y Marmiroli N. (2016). Molecular response of crop plants to engineered nanomaterials. Environ. Sci. Tech. 50 (13), 7198-7207. DOI: 10.1021/acs.est.6b01816

Peakall D.B. (1994) The role of biomarkers in environmental assessment. Ecotoxicology 3 (3), 157-160.

Pérez D.J., Menone M.L., Camadro E.L. y Moreno V.J. (2008). Genotoxicity evaluation of the insecticide endosulfan in the wetland macrophyte Bidens laevis L. Environ. Pollut. 153 (3), 695-698. DOI: 10.1016/j. envpol.2007.09.014

Pérez D.J., Lukaszewicz G., Menone M.L. y Camadro E.L. (2011). Sensitivity of Bidens laevis L. to mutagenic compounds. Use of chromosomal aberrations as biomarkers of genotoxicity. Environ.Pollut. 159 (1), 281- 286. DOI: 10.1016/j.envpol.2010.08.028

Pérez D.J., Lukaszewicz G., Menone M.L., Amé V.M. y Camadro E.L. (2014). Genetic and biochemical biomarkers in the macrophyte Bidens laevis L. exposed 
to a commercial formulation of endosulfan. Environ. Toxicol. 29 (9), 1063-1071. DOI: 10.1002/tox.21836 Pflugmacher S., Schröder P. y Sandermann H. (2000). Taxonomic distribution of plant glutathione-S-transferases acting on xenobiotics. Phytochemistry 54 (3), 267-273. DOI: $10.1016 / \mathrm{S} 0031-9422(00) 00116-3$

Pflugmacher S. (2004). Promotion of oxidative stress in the aquatic macrophyte Ceratophyllum demersum during biotransformation of the cyanobacterial toxin microcystin-LR. Aquat. Toxicol. 70 (3), 169-178. DOI: $10.1016 / j$.aquatox.2004.06.010

Ramachandran S., Rajendran N., Nandakumar R. y Venugopalan V.K. (1984). Effect of pesticides on photosynthesis and respiration of marine macrophytes. Aquat. Bot. 19 (3-4), 395-399.

DOI: 10.1016/0304-3770(84)90051-2

Sethunathan N., Megharaj M., Chen Z., Singh N., Kookana R.S. y Naidu R. (2002). Persistence of endosulfan and endosulfan sulfate in soil as affected by moisture regime and organic matter addition. B. Environ. Con. Tox. 68 (5), 725-731. DOI: 10.1007/s001280314

Silva Barni M.F. (2017). Evaluación de la biodisponibilidad de contaminantes orgánicos persistentes (COPs) a través del uso de muestreadores pasivos en la cuenca del Río Quequén Grande, Provincia de Buenos Aires. Tesis de Doctorado. Facultad de Ciencias Exactas y Naturales, Universidad Nacional de Mar del Plata, Mar del Plata, Argentina, 357 pp.

Sohn H.Y., Kwon C.S., Lee J.B. y Kim E. (2004). Induction of oxidative stress by endosulfan and protective effect of lipid-soluble antioxidants against endosulfaninduced oxidative damage. Toxicol. Lett. 151 (2), 357365. DOI: $10.1016 /$ j.toxlet.2004.03.004

Tasmin R., Shimasakia Y., Qiua X., Hondaa M., Tsuyamab M., Yamada N., Fukudad S. y Oshimaa Y. (2014a). Elevated temperatures and low nutrients decrease the toxicity of diuron for growth of the green alga Pseudokirchneriella subcapitata. Jpn. J. Environ. Toxicol. 17 (1), 1-10. DOI: 10.11403/jset.17.1
Tasmin R., Shimasaki Y., Tsuyama M., Qiu X., Khalil F., Okino N., Yamada N., Fukuda S., Kang I.J. y Oshima Y. (2014b). Elevated water temperature reduces the acute toxicity of the widely used herbicide diuron to a green alga, Pseudokirchneriella subcapitata. Environ. Sci. Pollut. Res. 21 (2), 1064-1070. DOI: 10.1007/s11356-013-1989-y

Turgut C., Grezichen A. y Fomin A. (2003). Toxicity of sulfonylurea herbicides to dicotyledonous macrophyte Myriophyllum aquaticum in a 14-day bioassay. Fresen. Environ. Bull.12 (6), 619-622.

Van Breusegem F., Vranová E., Dat J.F. e Inzé D. (2001). The role of active oxygen species in plant signal transduction. Plant Sci. 161 (3), 405-414.

DOI: $10.1016 / \mathrm{S} 0168-9452(01) 00452-6$

Van der Oost R., Beyer J. y Vermeulen N.P. (2003). Fish bioaccumulation and biomarkers in environmental risk assessment: A review. Environ. Toxicol. Pharmacol. 13 (2), 57-149. DOI: 10.1016/S1382-6689(02)00126-6

Vidal M.L., Bassères A. y Narbonne J.F. (2002). Seasonal variations of pollution biomarkers in two populations of Corbicula fluminea (Müller). Comp. Biochem. Phys. C 131 (2), 133-151.

DOI: $10.1016 / \mathrm{S} 1532-0456(01) 00291-5$

Viglizzo E.F., Frank F.C., Carreno L.V., Jobbagy E.G., Pereyra H., Clatt J., Pincen D. y Ricard M.F. (2011). Ecological and environmental footprint of 50 years of agricultural expansion in Argentina. Glob. Change Biol. 17 (2), 959-973.

DOI: 10.1111/j.1365-2486.2010.02293.x

Wendt-Rasch L., van den Brink P., Crum S. y Woin P. (2004). The effects of a pesticide mixture on aquatic ecosystems differing in trophic status: Responses of the macrophyte Myriophyllum spicatum and the periphytic algal community. Ecotox. Environ. Safe. 57 (3), 383398. DOI: 10.1016/j.ecoenv.2003.09.010

Zar J.H. (1996). Biostatistical analysis. $3^{\mathrm{a}}$ ed. Ediciones Internacionales Prentice-Hall, Upper Saddle River, Nueva Jersey, EUA, 662 pp. 